\title{
Detection of Brain Tumor for MRI using Hybrid Method Wavelet and Clustering Algorithm
}

\author{
Alyaa H. Ali \\ Asst. Professor Dept. Physics, \\ College of Science for women, \\ Baghdad University, Iraq
}

\author{
Kawther A.Khalaph \\ Msc. Student Dept. Physics, \\ College of Science for women, \\ Baghdad University,Iraq
}

\author{
Ihssan S.Nema \\ Asst. Professor Dept. \\ neurosurgery \\ college of medicine, \\ Alnahrain University, Iraq
}

\begin{abstract}
Automatic segmentation of brain tumor using computer analysis aided diagnosis in clinical practice but it is still a challenging task, especially when there are lesions needing to be outlined. In the applications of image-based diagnosis and computer-aided lesion detection, image segmentation is an important procedure .Features extracted from image analysis in companion with image segmentation algorithms are used to provide region-based information for clinical evaluation procedures. Brain tumor diagnosis is easy by using these medical equipments. The physician needs the correct measurement of the tumor area for the further treatment, this need to extract the abnormal part from the 2D MRI scan accurately and measure the region of interest. The HumanComputer interaction is helpful for this procedure. In this search the wavelet transformation is used as well as the $\mathrm{K}$ mean algorithm is used. the wavelet transformation is not sufficient to produce a good result for the brain tumor detection. so the K-mean clustering method with different classes gives best result.
\end{abstract}

\section{Keywords}

Brain Tumor, Wavelet, K-mean clustering, hybrid method.

\section{INTRODUCTION}

In order to get an information in the images we have to segment the images in different parts, to study the structure and features this help us to extracted region of interest [1]. In this search the MR images were used to classify the brain tumors, the medical images can be obtained from (CT) which is computed tomography, the (PET) which is the positron emission tomography and the (MRI) which is the magnetic resonance imaging. these type of images provides the human being with the diagnosis of various diseases [2]. the medical image segmentation is one of most difficult source of information of the human body which can help in reparative surgery, radiotherapy treatment planning, stereotactic neurosurgery Several new techniques have been used to improve the biomedical research. one of these techniques is the MRI. the MRI is a non-invasive technique for medical imaging that uses the magnetic field and pulses of radio waves. It gives better visualization of soft tissues of human body[2]. MRI has been early used to detect the brain tumors. the brain tumors which is the abnormal cell clusters and grow in the brain. the tumor can occurred in any person and any age. the tumor can be change from one person to the other and its effect is not the same it differ from one person the others. . Brain tumors appear at any location, in different image intensities, can have a variety of shapes and sizes. it can be malignant or benign [2]. Low grade gliomas and meningiomas which are benign tumors, and glioblastoma multiforme is a malignant tumor and represents the most common primary brain neoplasm. Benign brain tumors have a homogeneous structure and do not contain cancer cells. They may be either monitored radiologically or surgically destroyed completely, and they seldom grow back. Malignant brain tumors have a heterogeneous structure and contain cancer cells[3]. 1996 Gibbs et al. introduced a morphological edge detection technique combined with simple region growing to segment enhancing tumors on T1- weighted MRI data[4]. In 2001 Fletcher-Heath et al. proposed a combination of unsupervised classification with FCM and knowledge based image processing for segmentation of non-enhancing tumors [5]. In 2007 Dou et al. have proposed a fuzzy information fusion framework for brain tumor segmentation using T1-weighted, T2-weighted and PD images [6]. Baskaran et al. has proposed a method for texture based classification using binary decision tree[7]. In this search a hybrid technique which is the direct wavelet transformation (DWT) and K-mean algorithm. In the DWT we extracted the high pass image and then this image used as a new image to input in the K- mean algorithm for segmentation.

\section{Basic concept of work}

2.1 Wavelet based: the wavelet transformation is an important transformation which gives a description images that are limited in time and can describe edges and lines that are highly localized[1]. the wavelet based texture analysis have two important steps. The first one is the wavelet transform computation and the second one is the feature extraction [8]. Wavelet analysis is similar to the Fourier analysis. Wavelets are classified as linear transform that is capable of displaying the transformed output at multiple resolutions depending on the point of time/space and at the desired frequency. The wavelet transform was developed to overcome the shortcoming of the Short Time Fourier Transforms[9]. The wavelet transformation is important than the Fourier transformation because it gives good classification and segmentation of tumor from the brain MRI. By applying 2D two level wavelet decomposition of region of interest (ROI) is performed. Two dimensional (2-D) wavelet transform requires quad-tree filtering structure. It requires a set of high and low pass filters for the row data and another for the column data. The row and column data can be filtered separately one after another to yield four sets of coefficients i.e. approximate coefficients and three sets of detailed coefficients, one for each orientation (vertical, horizontal and diagonal). 2D wavelet transform uses multiresolutional analysis (Figure 1) on the signal by passing the input signal through the filter bank more than once[10]. The wavelet 
transform has good local character both in time domain and frequency domain. The low frequency coefficients reflect the outline information and the high frequency coefficients reflect the detail information and noise after a digital image was decomposed with wavelet transform. At the same time, the visual feeling of the general image is dependent on the lowfrequency information. On this point, we can do histogram equalization just in low frequency domain. So the detail can avoid being blurred and the noise can't be magnified if we just process the low frequency. [11] The 2-D wavelet decomposition of an image is performed by applying 1-D DWT along the rows of the image first, and, then, the results are decomposed along the columns.[12] This operation results in four decomposed sub band images referred to as low-low (LL),low-high (LH), high-low (HL), and high-high (HH).which result in four sub bands. In 2D wavelet decomposition the image is represented by one approximation and tree detail images, represented the low and high frequency

contents image respectively.
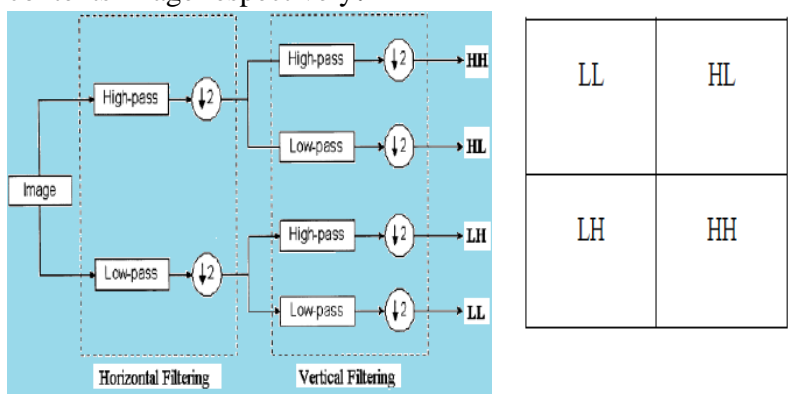

The result of 2-D

DWT decomposition

Figure (1) 2-DWavelet Transformation

\section{2 k-mean}

$\mathrm{K}$-means algorithm is one of important pattern recognition it's an unsupervised clustering algorithm which classifies the input data into multiple classes depending on the distance measured from each classes[1]. the $\mathrm{K}$-mean is a cluster analysis. It depend on partition (n) observer into $\mathrm{K}$ cluster with minimum distance and nearest mean) [13-14] . In KM algorithm, the classification depend on the measurement of the distance from the classes to cluster centroids every pixel is assigned to its closest cluster.

$$
\sum_{j=1}^{k} \sum_{i=1}^{N}\left\|I_{i}^{j}-c_{j}\right\|^{2}
$$

where $\left\|I_{i}^{j}-c_{j}\right\|^{2}$ is a chosen distance measure between a data point and the cluster centre $c_{j}$ an indicator of the distance of the $\mathrm{N}$ data points from their respective cluster centers. The generalized algorithm is composed of the following steps:

1. Place $\mathrm{K}$ points into the space represented by the objects that are being clustered.

2. Assign each object to the group that has the closest centroid.

3. When all objects have been assigned, recalculate the positions of the $\mathrm{K}$ centroids.

4.Repeat Steps 2 and 3 until the centroids no longer move. This produces a separation of the objects into groups from which the metric to be minimized can be calculated. [14] The grouping is done by minimizing the sum of squares of distances between data using Euclidean distance formula and the corresponding cluster centroid. In statistics and data mining, $k$-means clustering is a method of cluster analysis which aims to partition $n$ observations into $k$ clusters in which each observation belongs to the cluster with the nearest mean.[13-15] Figure( 2).

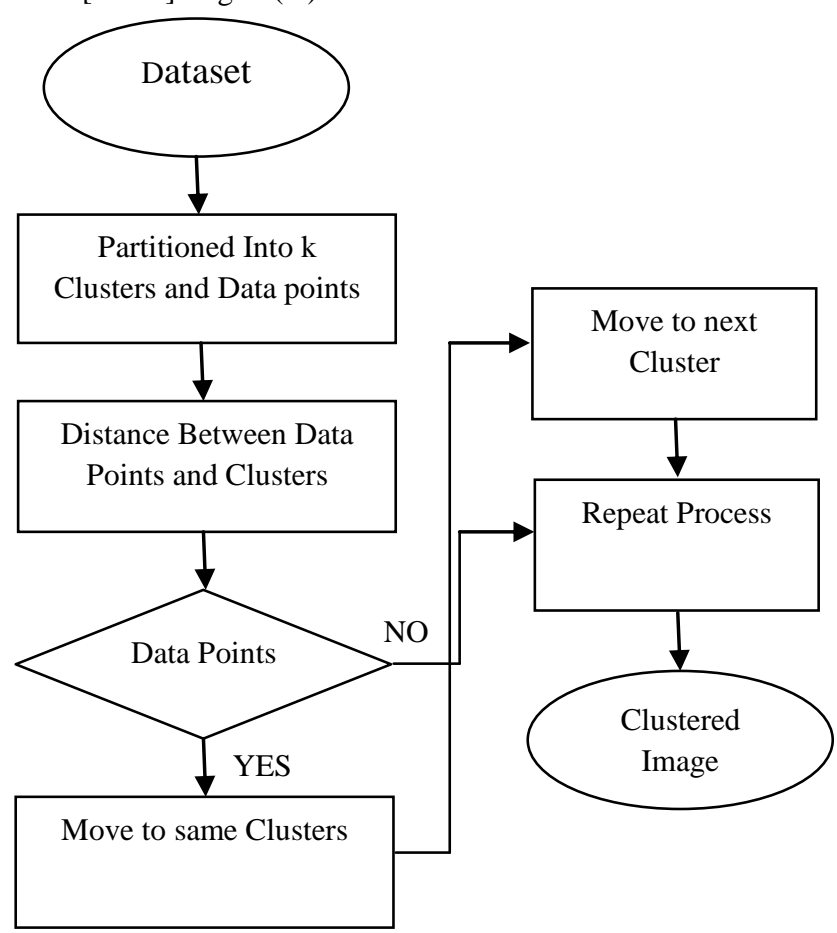

Figure(2). the flow chart of K-mean Algorithm

\section{The work and result}

In this research we use the wavelet transformation method and then the results used in k- mean clustering method we take different MR images and the number of clusters has been taken depending on the result. our images has been taken from Al-khadema Teaching Hospital as well as the images taken from internet.

\section{Discussion}

from the search one can see that the wavelet transformation is not sufficient to produce a good result for the brain tumor detection. so the K-mean clustering method with different classes gives best result. Table(1 to 6) shows the statistical features for the normal and abnormal part of the brain for different types of images. the entropy of abnormal part (tumor) we can see that the value for entropy increase compare with the part without tumor. this means that the numbers of gray level values increase so the energy will decrease because it inversely proportional to the entropy. the mean value which is the average value so it tell us something about the general brightness of the images, for the tumor part which appear as white region in the image the value of the mean increase compare to part without tumor. the median value which also increase for the tumor part as for the image without tumor. the stander deviation value which is also known as square root of the variance, tell us something about the contrast. it describe the spread in data, so the high contrast image will have a high variance and a low- contrast images will have low variance. for the tumor part the variance and the contrast increase. the homogeneity shows different changes sometimes increase and some time decrease so we cannot take 
it as a reference to classify the image if it has tumor or not. this can be shown in figures $(7,8,9)$.

The origin image

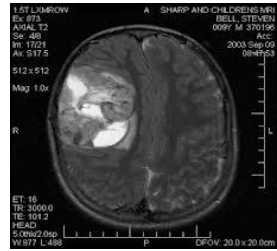

The origin image
After using wavelet transformation

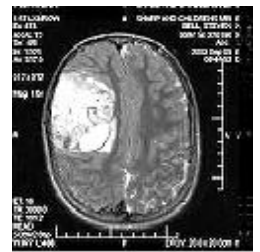

After using wavelet transformation

After using wavelet transformation

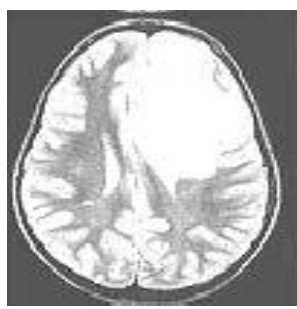

Using K-mean with cluster NO. $\mathrm{N}=6$

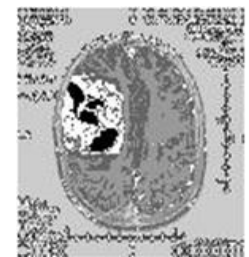

Using K-mean with cluster NO. $\mathrm{N}=5$

Using K-mean with cluster NO. $\mathrm{N}=4$
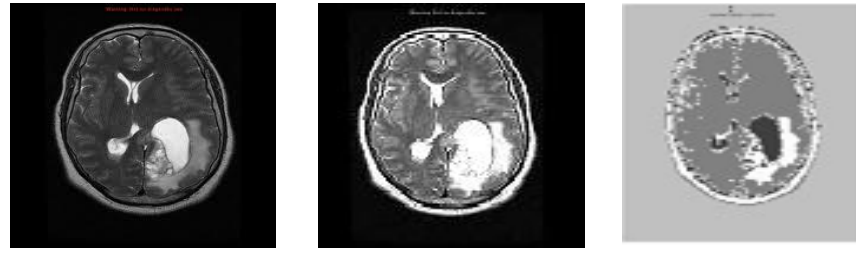

The origin image
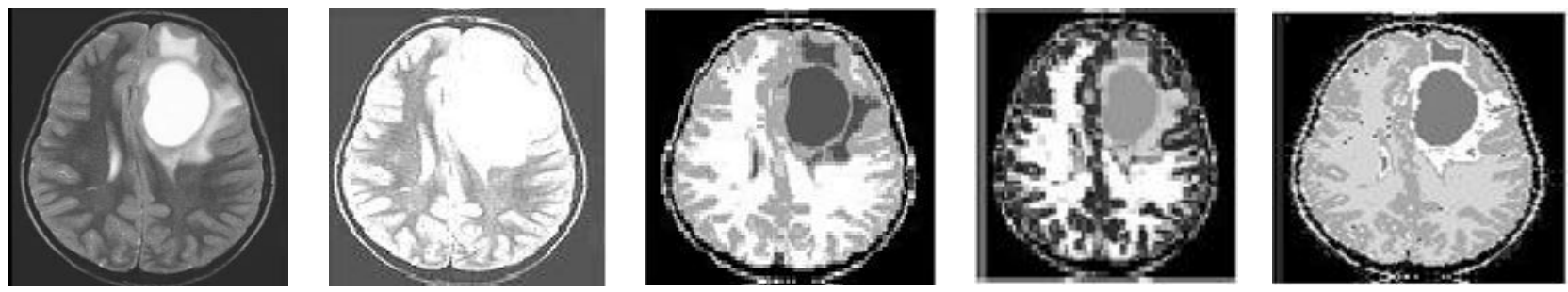

Figure(3-a) shows the MRI for brain tumor images taken from internet

After using wavelet transformation

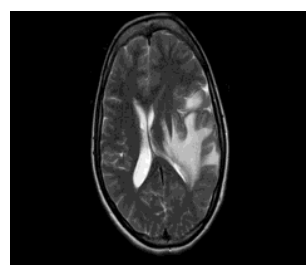

The origin image

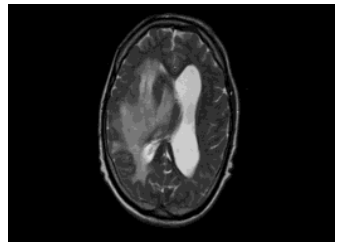
transformation
Using K-mean with cluster $\mathrm{NO} . \mathrm{N}=4$
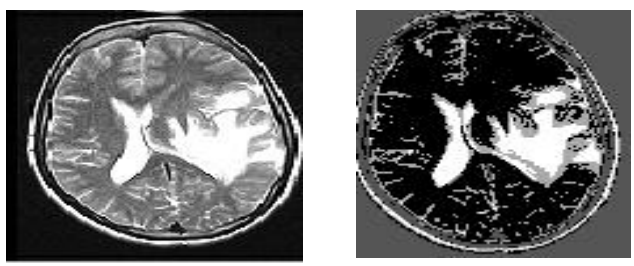
cluster $\mathrm{NO}$. $\mathrm{N}=5$

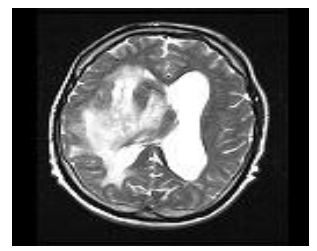
cluster $\mathrm{NO} . \mathrm{N}=5$

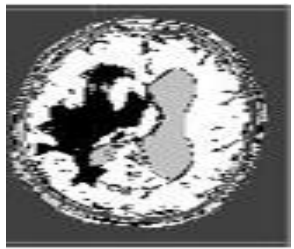

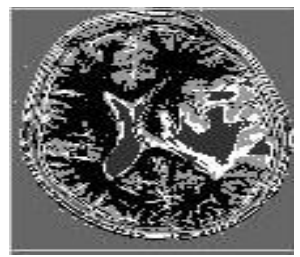

$\mathrm{N}=6$

$\mathrm{N}=6$

$\mathrm{N}=8$

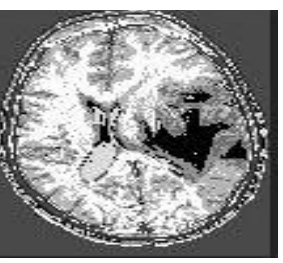

$\mathrm{N}=7$
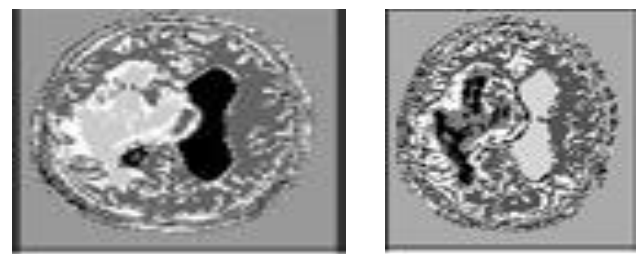

$\mathrm{N}=7$

Figure(3-b) shows the MRI for brain tumor images taken from Al Khadema Teaching Hospital . The origin image

After using wavelet transformation

\section{Using K-mean with} cluster $\mathrm{NO} . \mathrm{N}=3$
$\mathrm{N}=4$

$\mathrm{N}=5$ 

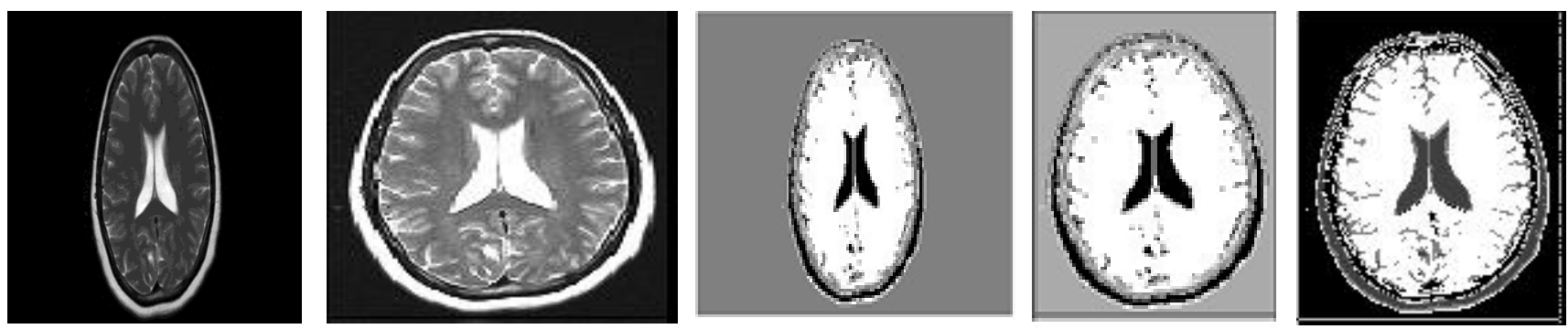

Figure(3-c) shows the MRI for normal brain images taken from Khadema Teaching Hospital.

Original image

Normal part Abnormal part

Original image

Normal part

Abnormal part
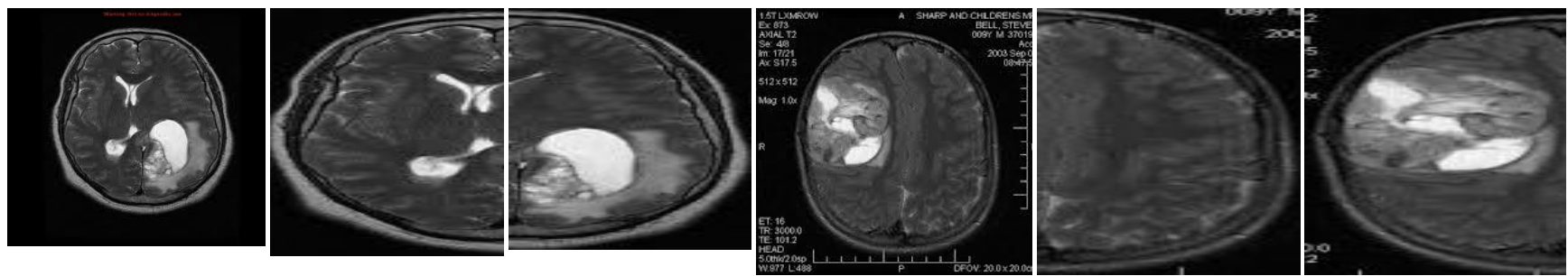

Figure(4) shows the original image with normal and abnormal part.

Original image

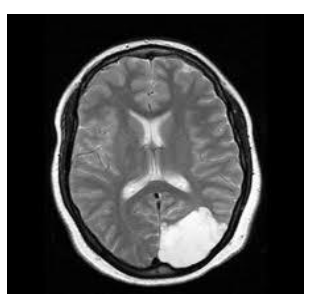

Normal image

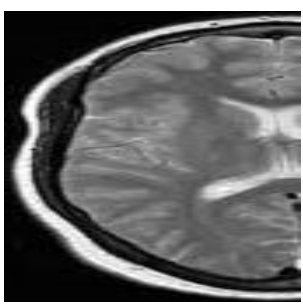

Figure(5) shows the original image with normal and abnormal part.

Figure(6) shows the original image with normal and abnormal part

Table(1) shows the statistical features for the normal part in figure(4).

\begin{tabular}{|l|l|l|l|l|l|l|l|l|l|}
\hline Entropy & Mean & Median & $\begin{array}{l}\text { standard } \\
\text { dev }\end{array}$ & $\begin{array}{l}\text { Median } \\
\text { abs.dev }\end{array}$ & $\begin{array}{l}\text { Mean } \\
\text { abs.dev. }\end{array}$ & Contrast & Correlation & Energy & Homogeneity \\
\hline 6.7210 & 57.76 & 54 & 44.38 & 21 & 31.2 & 0.5280 & 0.8435 & 0.1475 & 0.8422 \\
\hline
\end{tabular}

Table(2) shows the statistical features for the abnormal part in figure(4).

\begin{tabular}{|l|l|l|l|l|l|l|l|l|l|}
\hline Entropy & Mean & Median & standard dev & $\begin{array}{l}\text { Median } \\
\text { abs.dev }\end{array}$ & $\begin{array}{l}\text { Mean } \\
\text { abs.dev. }\end{array}$ & Contrast & Correlation & Energy & Homogeneity \\
\hline 6.9873 & 70.7 & 59 & 58.18 & 36 & 43.99 & 0.5334 & 0.92 & 0.1236 & 0.8571 \\
\hline
\end{tabular}


Table(3) shows the statistical features for the normal part in figure(5).

\begin{tabular}{|c|c|c|c|c|c|c|c|c|}
\hline Entropy & Mean & Median & $\begin{array}{c}\text { Standard } \\
\text { dev }\end{array}$ & $\begin{array}{c}\text { Median } \\
\text { abs.dev } \\
\text { abs.dev }\end{array}$ & $\begin{array}{c}\text { Mean } \\
\text { Contrast }\end{array}$ & Correlation & Entropy & Homogeneity \\
\hline 6.2859 & 50.38 & 55 & 26.33 & 14 & 20.67 & 0.2304 & 0.8331 & 0.2576 \\
\hline
\end{tabular}

Table(4) shows the statistical features for the abnormal part in figure(5).

\begin{tabular}{|l|c|c|c|c|c|c|c|c|c|}
\hline Entropy & Mean & Median & $\begin{array}{c}\text { standard } \\
\text { dev }\end{array}$ & $\begin{array}{c}\text { Median } \\
\text { abs.dev }\end{array}$ & $\begin{array}{c}\text { Mean } \\
\text { abs.dev. }\end{array}$ & Contrast & Correlation & Energy & Homogeneity \\
\hline 7.1610 & 70.43 & 63 & 55.71 & 33 & 41.03 & 0.2803 & 0.944 & 0.1596 & 0.8960 \\
\hline
\end{tabular}

Table(5) shows the statistical feature in the normal part in figure(6).

\begin{tabular}{|l|l|l|l|l|l|l|l|l|l|}
\hline Entropy & Mean & Median & $\begin{array}{l}\text { standard } \\
\text { dev }\end{array}$ & $\begin{array}{l}\text { Median } \\
\text { abs.dev }\end{array}$ & $\begin{array}{l}\text { Mean } \\
\text { abs.dev. }\end{array}$ & Contrast & Correlation & Energy & Homogeneity \\
\hline 7.1682 & 91.53 & 102 & 64.74 & 39 & 52.42 & 0.5605 & 0.9167 & 0.1460 & 0.8513 \\
\hline
\end{tabular}

Table(6) shows the statistical feature in the abnormal part in figure(6).

\begin{tabular}{|l|l|l|l|l|l|l|l|l|}
\hline Entropy & Median & $\begin{array}{l}\text { standard } \\
\text { dev }\end{array}$ & $\begin{array}{l}\text { Median } \\
\text { abs.dev }\end{array}$ & $\begin{array}{l}\text { Mean } \\
\text { abs.dev. }\end{array}$ & Contrast & Correlation & Energy & Homogeneity \\
\hline 7.2145 & 104 & 77.99 & 67 & 63.78 & 1.5930 & 0.9387 & 0.127 & 0.8580 \\
\hline
\end{tabular}

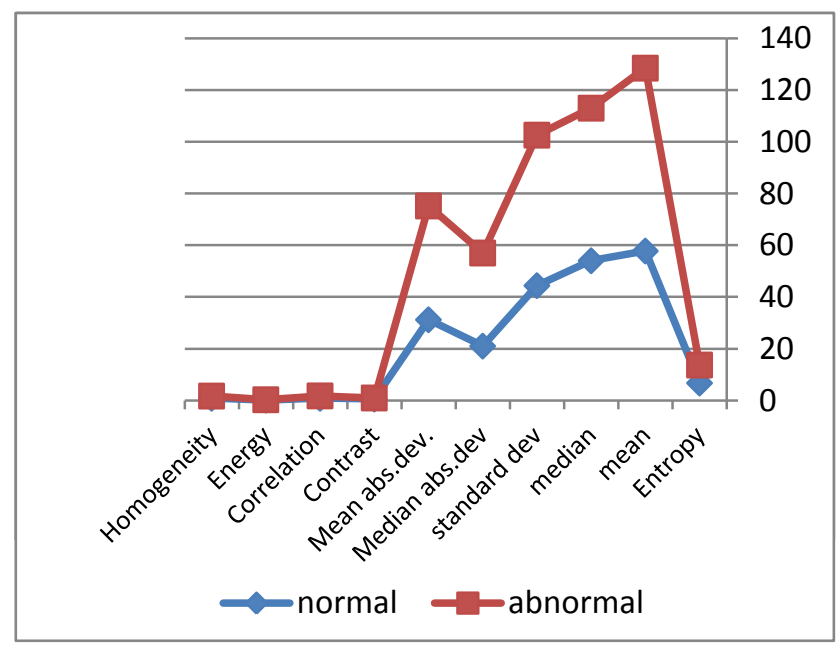

Figure(7) shows the graph of the statistical features in table $(1,2)$

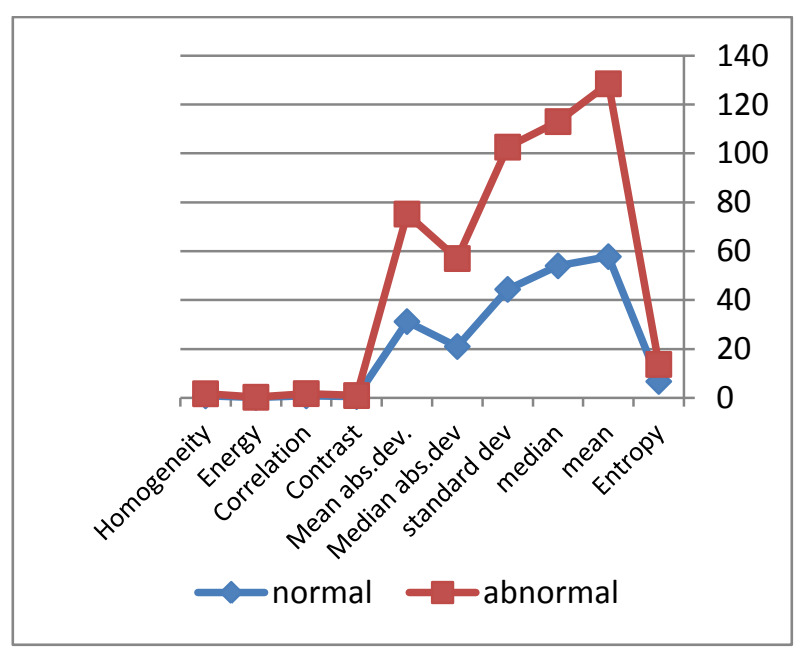

Figure(8) shows the graph of the statistical features in table $(3,4)$ 


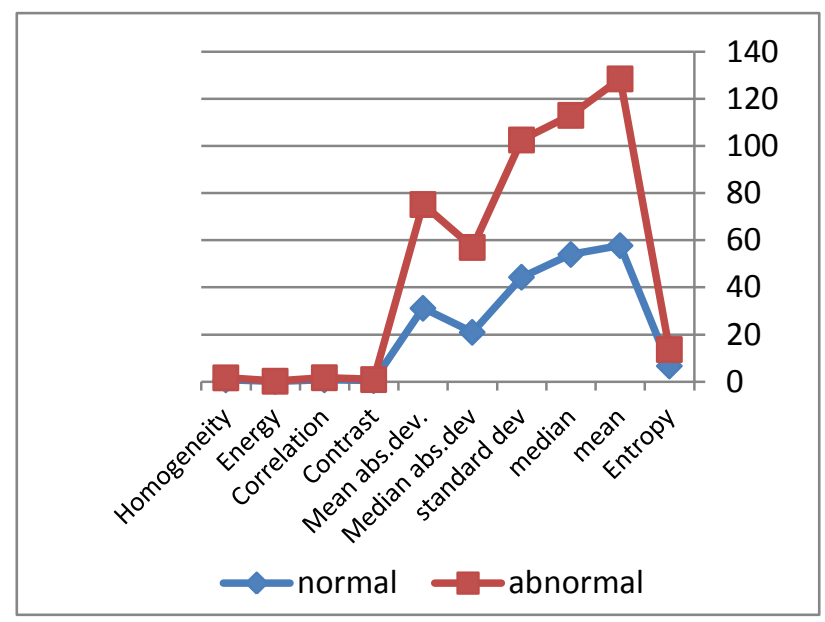

Figure(9) shows the graph of statistical features in table $(5,6)$.

\section{ACKNOWLEDGMENTS}

Our thanks to the experts who have contributed towards development of the template.

\section{REFERENCES}

[1] Rafael C. Gonzalez, Richard E. Wood, 2002. Digital Image Processing Personal Education.

[2] Shruti D., Avijit D. , Soumya K.. 2012 Application of Wavelet based K-means Algorithm in Mammogram Segmentation. International Journal of Computer Applications August 2012 52(15):15-19,. Published by Foundation of Computer Science, New York, USA.

[3] N.S.Zulpe, S.S.Chowhan, 2011. Statical Approach For MRI Brain Tumor Quantification", International Journal of Computer Applications December 2011 (0975-8887), vol.35-No.7,

[4] Gibbs,P, Buckley,D.L, and Black band. 1996, Tumor Volume Determinationfrom MRI by Morphological Segmentation". Phys Med Biol, 41(11): 2437-2446

[5] Fletcher-Heath, L. M, Hall, L. O and Goldgof. 2001, Automatic segmentation of non-enhancing brain tumors in magnetic resonance images". Artif Intell Med,21(13):43-63.

[6] Dou, W, Ruan, S, Chen, Y, Bloyet, D and Constans. 2007, A framework of fuzzy information fusion for segmentation of brain tumor tissues on MR images. Image and Vision Computing, Volume 25, Issue 2, February 2007.
[7] Baskaran.R, Deivamani.M, Kannan.A. 2004, A multiagent approach for texture basedclassification and retrieval MATBCR using binary decision tree. International journal of computing and information sciences, Vol. 2, No.1, 13-22.

[8] Tung-ying Wu and Sheng-fuu Lin. 2013, Segmentation of Parotid Lesions in CT Images using Wavelet-based Features. IJCA Special Issue on Recent Trends in

Pattern Recognition and Image Analysis RTPRIA(1):18-26, Published by Foundation of Computer Science, New York, USA, May 2013.

[9] Priya. M and C K Gobu. 2013, A Wavelet based Method for Text Segmentation in Color Images. International Journal of Computer Applications 69(3):14-17, Published by Foundation of Computer Science, New York, USA, May 2013.

[10] Vidya rajagopalan 2005, Neural Network Based Prognosis For Two dimensional Tumor-Like Growth". A thesis submitted to the Department of Electrical Engineering, The Florida State University, Summer Semester.

[11] Ramakrishnan and Murtaza Saadique Basha.2012 Color Image Enhancement based on Daubechies Wavelet and HIS Analysis. International Journal of Computer Applications 47(13):8-11, Published by Foundation of Computer Science, New York, USA, June 2012.

[12] Gajanand Gupta. 2011, Algorithm for Image Processing Using Improved Median Filter and Comparison of Mean, Median and Improved Median Filter. International Journal of Soft Computing and Engineering (IJSCE), ISSN: 22312307,Volume-1,Issue-5, November 2011.

[13] Ms.Chinki Chandhok, Mrs.Soni Chaturvedi and Dr.A.A Khurshid. 2012, AnApproach to Image Segmentation using K-means Clustering Algorithm. International Journal of Information Technology (IJIT), Volume - 1, Issue - 1, August 2012

[14] Neelofar Sohi, Lakhwinder Kaur and Savita Gupta .2012, Performance Improvement of Fuzzy C-mean Algorithm for Tumor Extraction in MR Brain Images .International Journal of Computer Applications 59(5):40-45, (December2012). Published by Foundation of Computer Science, New York, USA.

[15] Mary Praveena, Dr.IlaVennila.2010 , Optimization Fusion Approach for Image Segmentation Using KMeans Algorithm. International Journal of ComputerApplications (0975 - 8887)Volume 2 - No.7, June 2010. 